\title{
HOW TO DIFFERENTIATE FIBROSING PNEUMONITIS RELATED TO SSC AND COVID-19, THROUGH HRCT
}

Taila Furtado Ximenes ${ }^{1}$, Vítor Damasceno Leitão ${ }^{1}$, Ivan Henrique Ramos de Castro ${ }^{1}$, Marcos Lélio Máximo Pinho ${ }^{1}$, Sheila M de A Fontenele ${ }^{1, *}$

1. Hospital Regional Unimed Fortaleza, Fortaleza (CE), Brazil.

*Corresponding author: nilfort@uol.com.br

\section{BACKGROUND}

Differentiating pulmonary inflammatory impairment related to systemic sclerosis (SSC) from infectious forms, mainly viral, becomes challenging, especially when there is an overlap of clinical and tomographic findings of both diagnoses. Systemic sclerosis related to usual interstitial (UIP) and nonspecific pneumonitis (NSIP) shows initial high-resolution computed tomography (HRCT) with areas of attenuation in peripheral and bibasal ground glass, which progress indolently to traction bronchiectasis (honeycombing and interseptal thickening), usually associated with bilateral pleural effusion and lymph node enlargement, if an active inflammatory process. Viral pneumonias, including COVID-19, tend to develop, from the beginning, with peripheral and bilateral focal opacities and nodular ground glass, which can progress rapidly and dramatically to alveolar consolidation (with air bronchograms) and reticular opacities.

\section{CASE REPORT}

P1 - female, 86 years old, SSc with pulmonary fibrosis, systemic arterial hypertension (SAH) and diabetes mellitus (DM), presented in the emergency with episodic dry cough and progressive dyspnea for 3 days. She denied fever or flu-like symptoms. She was tachypneic (40 bpm), SPO2 76\% in room air, dehydrated and acrocyanotic; pulmonary auscultation with reduced breath sounds globally, fine bilateral crackles up to the right hemithorax apex and up to the middle third of the left. High-resolution computed tomography showed a change in the previous pattern, with reticular infiltrate with areas of ground glass, mosaic perfusion and air bronchograms in both lungs, lymph nodes dispersed in the mediastinal region. Conducted for sepsis with tazocin $4.5 \mathrm{~g} / \mathrm{FA}$ every $8 \mathrm{~h}$ and then teicoplanin 1,200 mg every $12 \mathrm{~h}$, and for COVID with azithromycin $500 \mathrm{mg}$ once daily and hydroxychloroquine $400 \mathrm{mg}$ every $12 \mathrm{~h}$, in addition to unfractionated heparin (UFH) 8,000 Ul every $8 \mathrm{~h}$ and methylprednisolone $250 \mathrm{mg}$ once daily. After orotracheal intubation (OIT) on $\mathrm{UCl}$, she needed vasoactive drugs and hemodialysis, and died after 10 days.

P2 - Female, 31 years old, SSc with pulmonary fibrosis, SAH and cardiopulmonary involvement. On the emergency room, she presented with flu-like symptoms for 4 days, use of azithromycin and ivermectin, evolving with progressive dyspnea for 1 day. She was feverish, tachypneic (40 bpm), SPO2 85\% in room air, dehydrated and acrocyanotic. High-resolution computed tomography showed a change in the previous pattern, with ground glass estimated at $50 \%$ of the parenchyma, in addition to extensive bilateral diffuse pulmonary opacities. ECHO with significant concentric left ventricular hypertrophy. Conducted for Severe acute respiratory syndrome with ceftriaxone, $2 \mathrm{~g}$ once daily, in addition to the COVID protocol. On the 5th day, she was transferred for ICU, because she needed OIT and vasoactive drugs, Tazocin and a methylprednisolone minipulse treatment. She evolved with superimposed infection and little anti-inflammatory response, and died of pulmonary hemorrhage.

\section{CONCLUSION}

In the face of SARS-CoV-2 pandemic, we must suspect and treat "the new on" pulmonary involvement of immunosuppressed scleroderma patients, with or without fibrosing pneumonitis, as by COVID-19, superimposed by bacterial infections and reactivation of the inflammatory process. Patients with SSc and sudden worsening of ventilatory parameters, suspected or confirmed by COVID-19, should be submitted to HRCT, without contrast initially, even in the mild phase and if chest X-ray is undetermined. Clinical discussion - rheumatologist, pulmonologist and radiologist, may be necessary. 
Table 1. Clinical-epidemiological aspects.

\begin{tabular}{ccc}
\hline Aspects & Patient 1 & Patient 2 \\
\hline Age (years) & Female & Female \\
\hline Comorbidities & 86 & 31 \\
Swab test for COVID-19 & $\begin{array}{c}\text { Systemic sclerosis, } \\
\text { pulmonary involvement } \\
\text { Systemic hypertension } \\
\text { Diabetes type II }\end{array}$ & $\begin{array}{c}\text { Systemic sclerosis, } \\
\text { pulmonary involvement } \\
\text { Systemic hypertension }\end{array}$ \\
\hline Initial symptoms & Not detected & Detected \\
\hline Symptom onset time (days) & Dyspnea/cough & Dyspnea/asthenia/fever \\
\hline O2 saturation at admission & 3 & 4 \\
\hline FR (irpm) at admission & 76 & 85 \\
\hline Length of stay in ICU (days) & 40 & 40 \\
\hline Tempo de internação na UTI (dias) & 9 & 8 \\
\hline
\end{tabular}

Table 2. Laboratory aspects.

\begin{tabular}{|c|c|c|c|c|}
\hline \multirow{2}{*}{ Aspects } & \multicolumn{2}{|c|}{ Patient 1} & \multicolumn{2}{|c|}{ Patient 2} \\
\hline & Admission & ICU entry & Admission & ICU entry \\
\hline Hemoglobin & $12.7 \mathrm{~g} / \mathrm{dL}$ & $11.8 \mathrm{~g} / \mathrm{dL}$ & $13.9 \mathrm{~g} / \mathrm{dL}$ & $11.1 \mathrm{~g} / \mathrm{dL}$ \\
\hline Leukocytes & $25,940 / \mu \mathrm{L}$ & $32,360 / \mu \mathrm{L}$ & 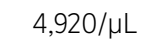 & $7,190 / \mu \mathrm{L}$ \\
\hline Lymphocytes & $1,038 / \mu \mathrm{L}$ & $971 / \mu \mathrm{L}$ & $940 / \mu \mathrm{L}$ & $503 / \mu \mathrm{L}$ \\
\hline Platelets & $362,000 / \mu \mathrm{L}$ & $372,000 / \mu \mathrm{L}$ & $156,000 / \mu \mathrm{L}$ & $245,000 / \mu \mathrm{L}$ \\
\hline CRP & $27.76 \mathrm{mg} / \mathrm{dL}$ & $18.80 \mathrm{mg} / \mathrm{dL}$ & $11.31 \mathrm{mg} / \mathrm{dL}$ & $16.50 \mathrm{mg} / \mathrm{dL}$ \\
\hline INR & 1.28 & 1.36 & - & 1.11 \\
\hline $\mathrm{LDH}$ & $531 \mathrm{UI} / \mathrm{L}$ & $397 \mathrm{UI} / \mathrm{L}$ & $427 \mathrm{UI} / \mathrm{L}$ & $392 \mathrm{UI} / \mathrm{L}$ \\
\hline D-Dimer & $3.34 \mu \mathrm{g} / \mathrm{mL}$ & $10.99 \mu \mathrm{g} / \mathrm{mL}$ & $0.54 \mu \mathrm{g} / \mathrm{mL}$ & $0.27 \mu \mathrm{g} / \mathrm{mL}$ \\
\hline Ferritin & $294.78 \mathrm{ng} / \mathrm{mL}$ & $332.01 \mathrm{ng} / \mathrm{mL}$ & - & $775.8 \mathrm{ng} / \mathrm{mL}$ \\
\hline Troponin & 59 ng/L & $97 \mathrm{ng} / \mathrm{L}$ & - & $5 \mathrm{ng} / \mathrm{L}$ \\
\hline Pro-BNP & $5,070.0 \mathrm{pg} / \mathrm{mL}$ & $28,537.0 \mathrm{pg} / \mathrm{mL}$ & - & $275.8 \mathrm{pg} / \mathrm{mL}$ \\
\hline Procalcitonin & $2.97 \mathrm{ng} / \mathrm{mL}$ & $4.94 \mathrm{ng} / \mathrm{mL}$ & - & $0.28 \mathrm{ng} / \mathrm{mL}$ \\
\hline Sodium & $126 \mathrm{mEq} / \mathrm{L}$ & $140 \mathrm{mEq} / \mathrm{L}$ & - & $141 \mathrm{mEq} / \mathrm{L}$ \\
\hline Potassium & $3.2 \mathrm{mEq} / \mathrm{L}$ & $4.0 \mathrm{mEq} / \mathrm{L}$ & $4.1 \mathrm{mEq} / \mathrm{L}$ & $5.0 \mathrm{mEq} / \mathrm{L}$ \\
\hline Creatinine & $0.89 \mathrm{mg} / \mathrm{dL}$ & $2.44 \mathrm{mg} / \mathrm{dL}$ & $1.48 \mathrm{mg} / \mathrm{dL}$ & $0.94 \mathrm{mg} / \mathrm{dL}$ \\
\hline Urea & $25 \mathrm{mg} / \mathrm{dL}$ & $82 \mathrm{mg} / \mathrm{dL}$ & $44 \mathrm{mg} / \mathrm{dL}$ & $38 \mathrm{mg} / \mathrm{dL}$ \\
\hline TGO & $29 \mathrm{U} / \mathrm{L}$ & $31 \mathrm{U} / \mathrm{L}$ & - & $23 \mathrm{U} / \mathrm{L}$ \\
\hline TGP & $18 \mathrm{U} / \mathrm{L}$ & $21 \mathrm{U} / \mathrm{L}$ & - & $20 \mathrm{U} / \mathrm{L}$ \\
\hline
\end{tabular}




\section{Pacient 1}

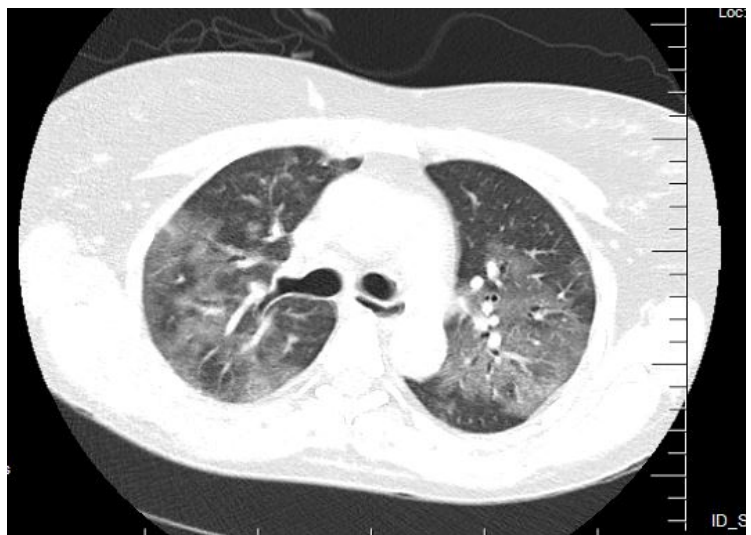

a) TCAR before COVID-19.

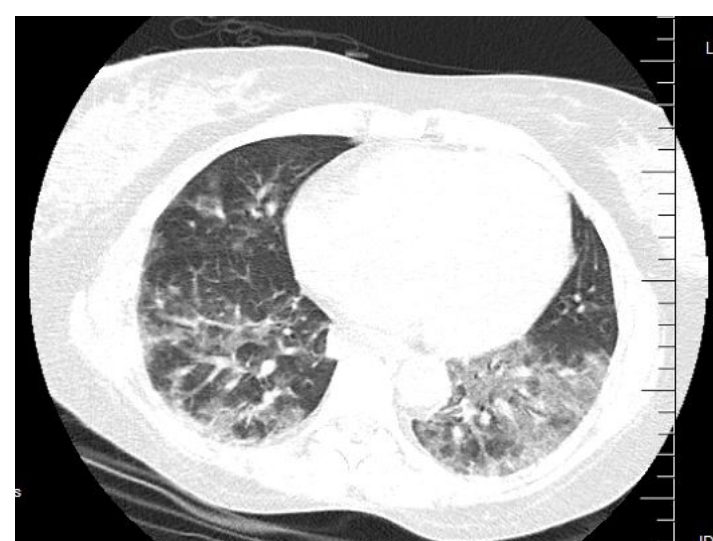

b) TCAR during COVID-19.

Figure 1. Evolution: reticular infiltrate with bilateral "frosted glass" areas of hypertransparency, mosaic perfusion and aerial bronchograms; important veiling on the right; foci of emphysematous lesions in superior lobes; numerous lymph nodes in the mediastinal region; unmeasured pulmonary parenchyma involvement.

Pacient 2

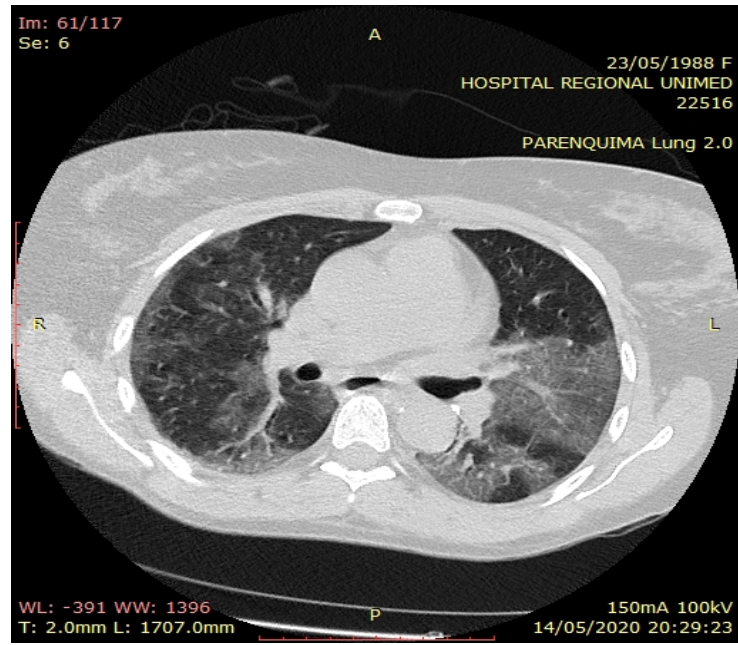

a) TCAR before COVID-19.

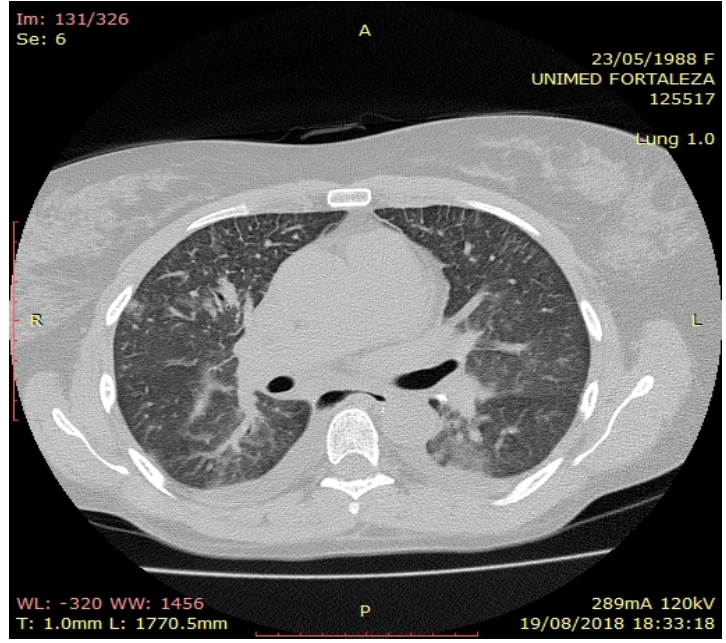

b) TCAR during COVID-19.

Figure 2. Evolution: multiple frosted glass lung opacities; thickening of interlobular septum in between; mosaic paving pattern with multifocal, bilateral, predominantly peripheral and posterior distribution; scattered bronchiectasis in the lower lobes; $50 \%$ pulmonary parenchyma involvement 